\title{
Validation of Advanced Constitutive Models for Accurate FE Modeling of TPU
}

\author{
Robert Eberlein ${ }^{1, *}$, Lucian Pasieka ${ }^{2}$, Dimosthenis Rizos ${ }^{3}$ \\ ${ }^{1}$ Institute of Mechanical Systems, Zurich University of Applied Sciences, Winterthur, Switzerland \\ ${ }^{2,3}$ Eugen Seitz AG, Wetzikon, Switzerland
}

*Corresponding author: E-mail: ebei@zhaw.ch

Received: 16 April 2019, Revised: 22 May 2019 and Accepted: 25 May 2019

DOI: $10.5185 /$ amlett.2019.0031

www.vbripress.com/aml

\begin{abstract}
Thermoplastic polyurethanes (TPU) have become preferred materials for demanding high strain rate applications in many industries throughout past years. Due to their comparatively high abrasion resistance and toughness, TPU materials form an excellent fit for critical components sustaining high pressures in combination with harsh ambient conditions. This presentation illustrates a comparatively new field of critical applications for TPU components. While the operational pressures remain rather moderate at maximum 50 bar, challenges arise from high-frequency, cyclic loading conditions. In order to design robust dynamic TPU components, two main tasks must be accomplished: (i) visco-elastic-plastic material modeling and parameter identification, and (ii) material validation under realistic dynamic loading conditions on system level by means of advanced finite element (FE) simulations. This article puts (i) emphasis on the material calibration process and (ii) specifically demonstrates material validation on system level for selected TPU materials. In this context strain rate dependency of various TPU grades is discussed, which illustrates deficiencies of classical material modeling techniques available in commercial finite element software versus advanced nonlinear models. Eventually, recommendations are provided for an efficient but also accurate material calibration process of solid TPU materials that can significantly enhance product innovation processes. Copyright (O VBRI Press.
\end{abstract}

Keywords: TPU, system validation, material calibration, FE simulation.

\section{Introduction}

Starting point of the current research was developing high endurance TPU components subjected to high strain rate applications The strategy considered for reaching this goal was to select high quality materials available in the market and design them under dynamic loading conditions. Doing that the major scientific/technical challenge is the nonlinear nature (visco-elastic-plastic) of the TPU materials to be used for such critical components. On top of that there is not a standardized engineering practice to design polymeric components under highly dynamic loading conditions. The innovation content of the current article aims at this and eventually allows the involved industry partner for the development of new innovative products. A modular approach to perform so is to develop highly sophisticated systems, which, when systematically combined and tailored to fit the individual customer needs, deliver reliably the requested functionality and form a distinct competitive advantage.

For the aforementioned reasons the major focus of this article is the validation of TPU components under highly dynamic, i.e. high strain rate, cyclic loading conditions. Towards that direction a finite element (FE) based simulation tool is required for designing purposes. This tool must take into consideration (i) the thermomechanical properties of the specific polymeric materials used under high amplitude of strains and strain rates at different temperatures, (ii) the coupling between the dynamic loading conditions and the nonlinear thermomechanical properties of the specific polymeric material. Such a tool is not generally available as "ready to be used module" in commercial FE codes/software (e.g. Ansys and Abaqus). In fact, there are three major shortcomings: (i) material data for polymers at high strain rates is not easily accessible, (ii) calibration of dynamic material data for suitable visco-elastic-plastic constitutive models is not (fully) integrated and (iii) only limited (linear visco-elastic) modeling techniques are available in most cases.

The investigations presented in this article address all three shortcomings related to current commercial FE software packages. By means of thermoplastic polyurethane elastomers (TPU) it is shown that depending on their specific strain rate dependency reasonable solutions can partially be found with classical concepts (CC) but for other materials advanced concepts (AC) are required as indicated in Fig. 1. 


\section{TPU Material Modeling}

Classical Concept (CC):

Linear Visco-Elasticity

Fig. 1. TPU material modeling concepts.

The specific novelty of this paper is the presentation of a customized experimental testing strategy that allows system validation by accurately quantifying $\mathrm{CC}$ vs $\mathrm{AC}$ FE models of selected TPUs under highly dynamic loading conditions. In addition, this paper outlines the constitutive modeling strategies for both TPU material calibration concepts that close the gaps of current commercial FE software packages. Furthermore, recommendations are provided for efficient FE modeling without sacrificing modeling accuracy. The results presented in this paper eventually lay ground for designing highly dynamic system applications.

\section{Experimental}

The effectiveness of CC and AC model approaches is assessed via the cross-validaiton principle (tests used for validation are different to these used for material model calibration). To achieve so, a dedicated test-bench has been used to measure "implicitly" the high-strain-rate behavior of the TPU under test (Fig. 2). In parallel, this test-bench system is FE-based modelled, employing each of the aforementioned material models, respectively. Via comparing the simulated to the experimentally obtained counterparts, the ability of each corresponding material model to reproduce in good terms the real high-strain-rate behavior of the TPU under test can be independently assessed.

To execute a high-strain-rate test on the test-bench, the TPU under test is firmly clamped on the base (Fig. 2). The lightweight metallic piston (mass < 32g), while lying on its "starting position" [Fig. 2 (a)], is getting ejected towards the TPU under test. When the piston establishes contact with the TPU under test, it has velocity $v_{l}$ [Fig. 2 (b)]. During contact, the TPU under test is deformed at high-strain-rate due to the momentum of the piston. Finally, the piston bounces backward with velocity $v_{2}$.

During the test, the vertical movement and the proper alignment of the piston is achieved by two low friction bearings (friction force $<1 \mathrm{~N}$ - Fig. 2). Right before, during and right after the contact of the piston with the TPU under test, three external forces act on the piston. These are the friction $(<1 \mathrm{~N})$, its weight $(<0.5 \mathrm{~N})$ and the reaction force from the TPU under test. The first two are negligible compared to the latter (to establish boundary conditions for the FE model simulation later). Practically speaking the only significant force acting on the piston during the contact is the reaction force.Its counterpart is responsible for the high-strain-rate deformation on TPU under test.

The piston, while moving: Fig. 2 (a) $\rightarrow$ Fig. 2 (b), is monitored by a Polytec laser vibrometer at point $\mathrm{A}$.
Due to this, the displacement and velocity of the piston (Fig. 2) are both measurable and readily available as acquired signals (both sampled at $1 \mathrm{MHz}$ ).

The measured velocity of the piston during the contact is an "implicit" expression of the high-strain-rate deformation of the TPU under test. Therefore, the comparison of the measured velocity with the FE-based simulated counterpart provides an independent highlevel criterion to assess the ability of each of the underlying material models to capture the high-strainrate behavior of the TPU material.
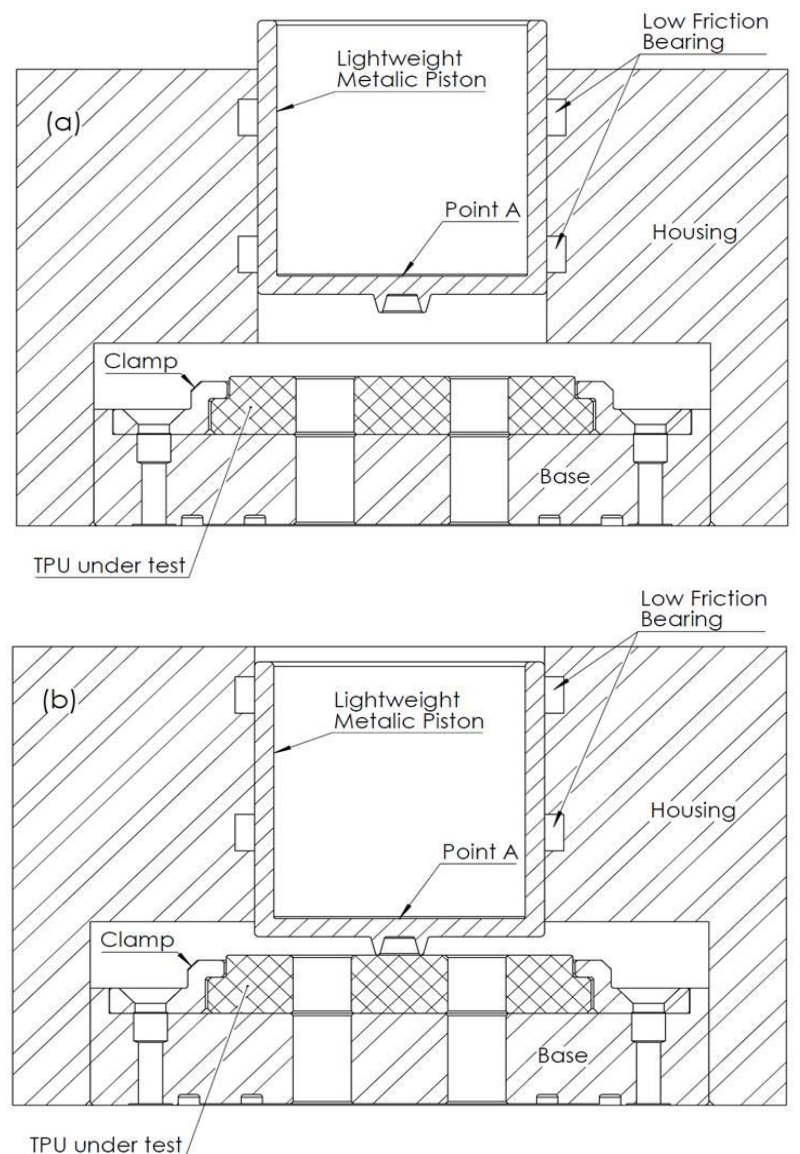

Fig. 2. Schematic sketch of the test-bench for capturing "implicitly" the high-strain-rate behavior of the TPU under test: (a) Starting position: the lightweight metallic piston is about to get ejected towards the TPU specimen; (b) Contact position: the lightweight metallic piston contacts the TPU under test with velocity $v_{l}$; after the pertinent deformation of the TPU material the piston bounces backwards at $v_{2}$ velocity.

\section{Results and discussion}

Before discussing TPU material calibration and validation results, the underlying $\mathrm{CC}$ and $\mathrm{AC}$ material models are briefly outlined.

\section{CC model description}

Polymeric materials are mostly characterized by elastic and viscous (time dependent) responses when loaded. The simplest way to model this behavior is through linear visco-elasticity. The underlying theoretical framework has been applied for years and the interested reader is referred to standard literature on the subject [5, 7, 12]. 
The stress response of a stress relaxation experiment for a visco-elastic material can be written in integral form:

$$
\sigma(t)=\int_{-\infty}^{t} E_{\mathrm{R}}(t-\tau) d \varepsilon(t)=\int_{-\infty}^{t} E_{\mathrm{R}}(t-\tau) \frac{d \varepsilon(\tau)}{d \tau} d \tau .
$$

This equation is integrated in parts to:

$$
\sigma(t)=E_{0} \varepsilon(t)-\int_{0}^{t} \dot{g}_{R}(t-\tau) E_{0} \varepsilon(\tau) d \tau,
$$

where the normalized relaxation modulus $g_{\mathrm{R}}(t)=E_{\mathrm{R}}(t) / E_{0}$ has been introduced. The instantaneous modulus $E_{0}$ is given by $E_{0}=\lim _{t \rightarrow 0} E(t)$. By means of a number of parallel Maxwell models (i.e. a rheological model consisting of a linear spring and a linear damper in series) the stress relaxation modulus can be written as:

$$
E_{\mathrm{R}}(t)=E_{0}-\sum_{i=1}^{N} E_{i} e^{-t / \tau_{i}}
$$

This representation is also called Prony series relaxation modulus function. The Prony series terms can be determined through time dependent experiments like creep, stress relaxation or dynamic mechanical analysis (DMA). For large elastic strains a hyperelastic stress function can be introduced:

$$
\sigma_{\text {hyp }}(\varepsilon)=\sigma_{\text {hyp }}(\varepsilon(t)) \equiv E_{0} \varepsilon(t)
$$

The resulting large strain linear visco-elasticity (CC) material model is available in all major commercial FE programs. These programs do often also include calibration of hyperelastic material parameters and Prony series terms. The major limitation of a CC material model is its purely linear viscous material behavior, though. For most elastomer-like materials CC material modeling is sufficiently accurate but for TPUs there can be severe shortcomings as shown below.

\section{AC model description}

One of the first generalizations of the CC model was given by the Bergstrom-Boyce (BB) model [3]. It is an advanced constitutive model for predicting the nonlinear time-dependent, large strain behavior of elastomer-like materials. The BB model states that the true response of an elastomer can be represented by two parallel networks $\mathrm{A}$ and B. Network A is a nonlinear hyperelastic network comparable to the $\mathrm{CC}$ model but network $\mathrm{B}$ consists of a nonlinear hyperelastic component in series with a nonlinear viscoelastic flow element (i.e. a nonlinear Maxwell element). A further expansion of this concept is given by the Three Network Model (TNM), which is denoted as the AC model throughout this paper. A detailed description of the constitutive framework for the TNM can be found in [4]. The TNM was specifically developed for thermoplastic materials. As demonstrated throughout this paper it also works very well for elastomer-like TPU materials.
The rheological representation of the AC material model (TNM) is given by three parallel networks: two nonlinear spring/damper networks $\mathrm{A}$ and $\mathrm{B}$ and $\mathrm{a}$ nonlinear spring network $C$. Using this framework, the total Cauchy stress in the system is given by $\boldsymbol{\sigma}=\boldsymbol{\sigma}_{A}+\boldsymbol{\sigma}_{B}+\boldsymbol{\sigma}_{C}$. The governing equations for the stress in each element of the model are as follows:

Cauchy stress in network A:

$$
\boldsymbol{\sigma}_{A}=\frac{\mu_{A}}{J_{A}^{e} \overline{\lambda_{A}^{e^{*}}}}\left[1+\frac{\theta-\theta_{0}}{\hat{\theta}}\right] \frac{\mathcal{L}^{-1}\left(\overline{\lambda_{A}^{e^{*}}} / \lambda_{L}\right)}{\mathcal{L}^{-1}\left(1 / \lambda_{L}\right)} \operatorname{dev}\left[\mathbf{b}_{A}^{e^{*}}\right]+\kappa\left(J_{A}^{e}-1\right) \mathbf{1} .
$$

Cauchy stress in network $\mathrm{B}$ follows by swapping A to $\mathrm{B}$. Cauchy stress in network $\mathrm{C}$ reads as:

$$
\begin{aligned}
\boldsymbol{\sigma}_{C}=\frac{1}{1+q}\left\{\frac{\mu_{C}}{J \lambda_{\text {chain }}}\left[1+\frac{\theta-\theta_{0}}{\hat{\theta}}\right] \frac{\mathcal{L}^{-1}\left(\frac{\lambda_{\text {chain }}}{\lambda_{L}}\right)}{\mathcal{L}^{-1}\left(\frac{1}{\lambda_{L}}\right)} \operatorname{dev}\left[\mathbf{b}^{*}\right]\right. \\
\left.+\kappa(J-1) \mathbf{1}+q \frac{\mu_{c}}{J}\left[I_{1}^{*} \mathbf{b}^{*}-\frac{2 I_{2}^{*}}{3} \mathbf{I}-\left(\mathbf{b}^{*}\right)^{2}\right]\right\} .
\end{aligned}
$$

Without going into further details here, the model parameters and assumptions can be found in literature $[1,4]$.

Extensions of the presented AC material modeling were recently published in $[\mathbf{8}, \mathbf{9}]$ for anisotropic finite strain viscoelasticity. The anisotropic models proposed account for nonlinear stress response in fiber-reinforced polymers and will be validated at a later point in time for suitable industrial applications.

\section{TPU material calibration}

There are various commercial software packages that can be used to calibrate a material model from experimental data and, as already mentioned above, most FE programs contain some functionality for material model calibration (e.g. CC material model). None of the major commercial FE programs, however, include a general-purpose material model calibration tool, or even tools for all the material models that are included in their material libraries. In fact, the BB model among others is implemented in FE programs like e.g. Abaqus or Ansys but a straightforward calibration is not possible there. One approach that is sometimes used to overcome this problem is to rely on trial-and-error techniques. Nevertheless, this approach is very time-consuming and, in many cases does not yield satisfactory results due to the strong nonlinearity of the involved material parameters.

For the AC model (TNM) used in this paper, TPU material parameters are calibrated to experimental test data at high strain rates. In contrast, the $\mathrm{CC}$ model parameters are calibrated at low strain rates based on experimental data from cyclic DMA measurements up to $50 \mathrm{~Hz}$ that form the current industry standard. Due to assumption of time-temperature equivalence for the $\mathrm{CC}$ model, the experimental data at various temperatures for the storage modulus is shifted in the frequency domain 
in order to form a so-called master curve according to [13], against which the Prony series terms can be calibrated. Thus, the CC model is also made accessible for high strain rate applications. In addition, quasi-static uniaxial, equibiaxial and planar tension test data form the basis for calibrating the hyperelastic material parameters of the CC model. For the current TPU materials the 3-term Ogden model provides excellent fits of the experimental data (refer to [11] for a detailed account on hyperelastic material modeling). The CC model calibration is eventually done in a commercial $\mathrm{FE}$ program.

In general, the Nelder-Mead simplex optimization method [10] can optimize the material parameters to best fit experimental data. The Nelder-Mead method is appropriate for material parameter extraction because of its robustness. It can be addressed by e.g. Matlab as builtin implementation. Another option is using commercial software packages, like e.g. MCalibration by Veryst Engineering, Needham, MA. Alternative algorithms like genetic algorithms can also be employed [6].

(a)

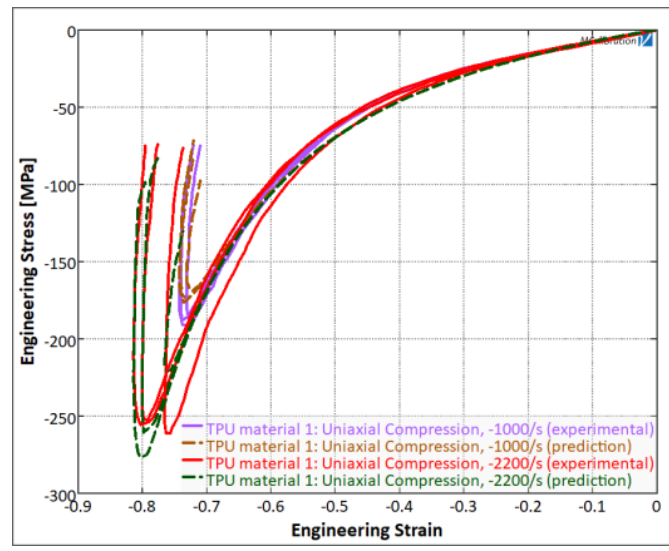

(b)

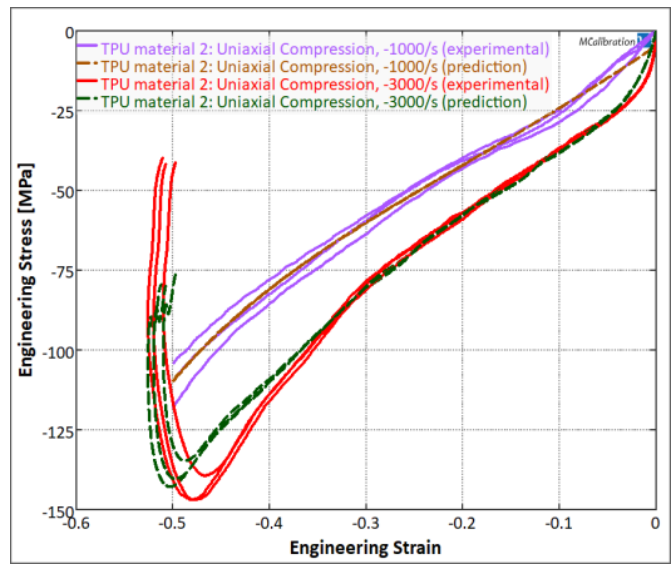

Fig. 3. Best AC model fit of high strain rate experimental data for (a) TPU material 1 and (b) TPU material 2 at room temperature.

Fig. 3 demonstrates the AC model material calibration procedure with MCalibration for two selected TPU materials. It depicts the predicted stress strain curves in compression after material parameter extraction at two different strain rates per TPU material. For both TPU materials under test, three material samples were tested per strain rate, which lead to individual predictions for each sample. As can be observed from the experimental data, TPU material 1 is only weakly strain rate dependent, whereas TPU material 2 shows a strong strain rate dependency. This fact raises the expectation that the $\mathrm{CC}$ model could potentially suffice for accurately mapping TPU material 1 behavior in real applications, whereas for TPU material 2, the AC model is required, instead.

\section{TPU material validation}

The calibrated CC and AC material models are compared with validation test data obtained from the test setup reported in the Experimental section. In Fig. 4, the dynamic response of TPU materials under test is reported in terms of velocity over time for a piston as illustrated in Fig. 2. The velocity-time curves are normalized with respect to the ingoing piston velocity $v_{l}$ just before impact. As described in the explanations for the validation test, $v_{l}$ is set as initial condition in the FE simulations. During FE simulations the piston runs freely until it hits the TPU under test. Its transient behavior depends on its mass inertia and triggered by it, the dynamic response of the TPU under test. The deformations of the metallic piston are negligible in this context. Such a validation scheme forms a very stringent challenge for the discussed constitutive models, since they need to replicate the experimental findings in a well-defined but also unconfined high strain rate application.

(a)

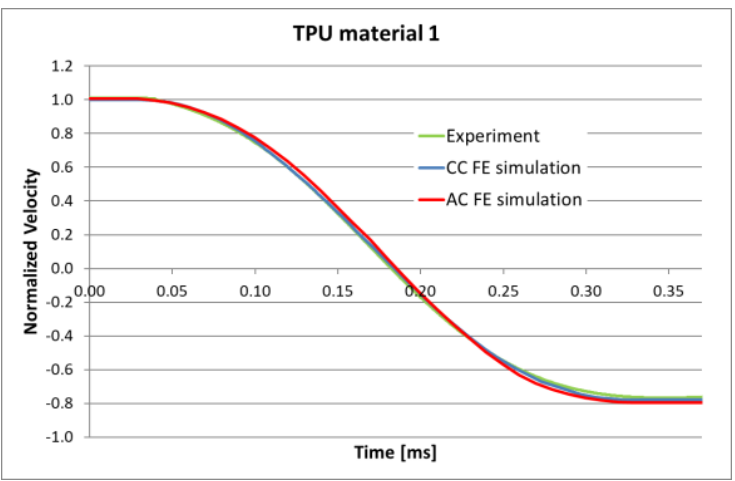

(b)

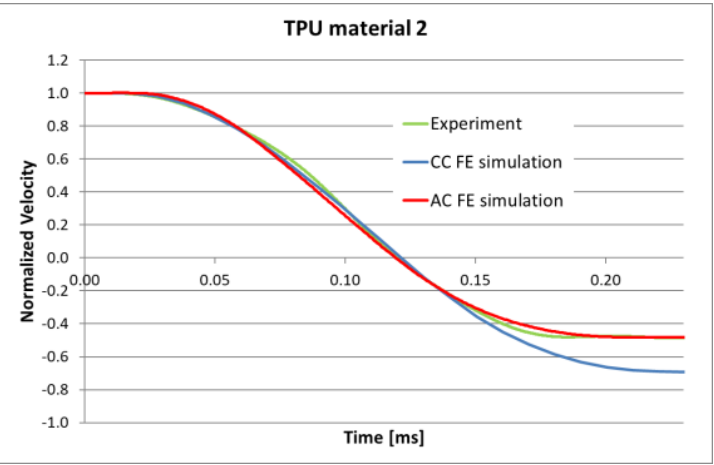

Fig. 4. Dynamic response of (a) TPU material 1 and (b) TPU material 2 at room temperature. 
TPU material 1 has proven to be only weakly strain rate dependent at room temperature. As reckoned before validation test results can be matched by a CC model based FE simulation as accurately as they can be by an AC model approach [Fig. 4 (a)]. This fact proves that the assumption of a time-temperature equivalence is valid for this specific TPU material. On the other hand, the FE simulations for TPU material 2 show a significantly different behavior. During simulation this material can be accurately represented by the AC model, only. The predicted outgoing piston velocity $v_{2}$ of the $\mathrm{CC}$ model simulation overestimates the experimental findings by $44 \%$, whereas the AC model yields very good results compared with experiment [Fig. 4 (b)].

These results clearly illustrate the limitations of CC material modeling. If highly strain rate dependent materials have to be simulated at high strain rates by FE, CC model based linear visco-elasticity is not good enough to accurately determine material damping properties. In those cases, AC models are mandatory for receiving realistic simulation results. Here, it is important to note that TPUs are elastomer-like materials from a mechanical point of view and many $\mathrm{FE}$ simulation engineers still prefer CC modeling based on e.g. material calibration obtained from standardized DMA test results. In addition, the current findings are also confirmed for TPU material validations apart from room temperature. At elevated temperatures the illustrated effects become even more prominent, since most TPUs show increasing strain rate dependency at rising temperatures.

AC type models can only capture the viscous time dependent response of a polymeric material, if the material is tested at different strain rates, preferably very close to the actual applications. As an example, Fig. 5 illustrates the compressive strain distribution in a TPU material 2 specimen for the AC model FE simulation depicted in Fig. 4 (b). The maximum strain value of about $38 \%$ lies well within the testing range of TPU material 2 for different strain rates as reported in Fig. 3 (b). Due to the ring structure of the piston contact zone (see Fig. 2), the strain distribution in Fig. 5 is unsymmetric under the piston tip.

Finally, it must be mentioned that AC type models can be effectively calibrated as demonstrated in this paper but this process is not yet integrated in commercial FE programs. This might also form a threshold for many FE simulation engineers to apply these models today. Another important aspect for practical use of AC type models is the manifold of experimental material data used for calibration. As more experimental material data is acquired as more elaborate it becomes to perform an accurate material parameter extraction. Therefore, the experimental material data acquisition should always be well prepared and restricted to a physically meaningful minimum amount of tests as shown for the current TPU examples. This restriction does not only apply to various strain rates but also to the actual strain and temperature ranges.

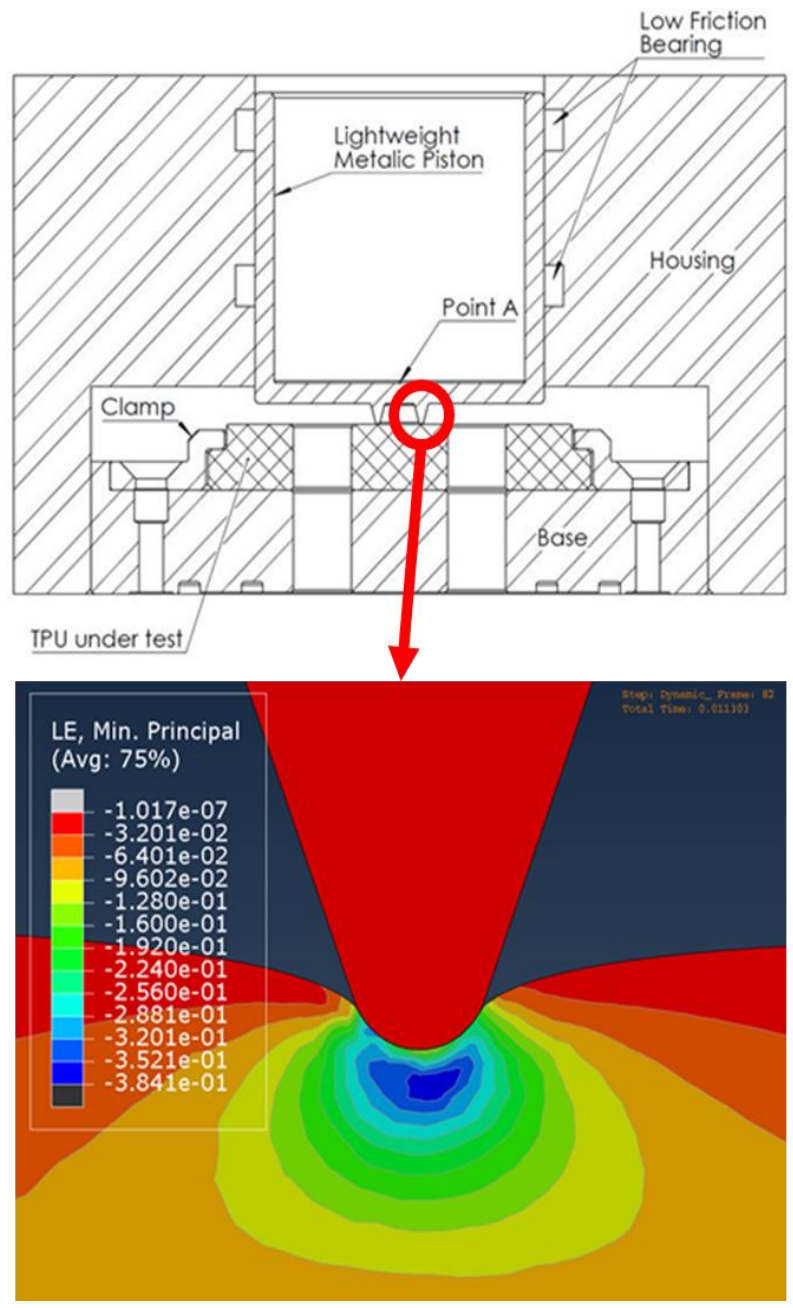

Fig. 5. Compressive strain distribution in AC model FE simulation for TPU material 2 specimen at time of maximum piston penetration.

\section{Conclusion}

The current paper demonstrates a validation scheme for TPU material models that properly accounts for viscoelastic-plastic strains at high strain rates (AC model). Limitations of $\mathrm{CC}$ modeling are addressed and prerequisites for efficient usage of $\mathrm{AC}$ models are discussed. By means of employing the right methodology in material data acquisition, material data calibration and subsequent FE modeling, TPU material behavior can be accurately and efficiently determined for practical applications. Thus, the limitations of current commercial FE software tools are eliminated. One very important finding is that FE modeling accuracy for TPU materials can be brought to almost arbitrarily high levels by focusing on material data acquisition that is dominant for a specific application. This means that advanced visco-elastic-plastic material models must be calibrated in proper strain, strain rate and temperature domains that are close to targeted applications. In general, a comprehensive fit across very broad strain rate and temperature ranges is not beneficial, even though the underlying constitutive theories would allow doing that. In those cases, the approximation quality of material 
parameters and the robustness of the resulting FE models are jeopardized.

In future, the presented AC material modeling will be used for validation of other polymers, including thermoplastic materials at high strain rates. First investigations in that direction are very promising. On top of that co-workers of the corresponding author [2] have recently shown that the presented AC type modeling can favorably be employed for accurate FE modeling of 3D-printed polymeric auxetic re-entrant structures. In contrast to analytical or linear elastic modeling techniques as reported e.g. in [14], the AC type modeling approach is very accurate and additionally valid during unloading of auxetic re-entrant structures.

Eventually, the findings of this paper will be directly deployed for designing dynamic system applications. Due to the high FE model accuracy, AC type material models will allow reducing very time-consuming (one year plus) endurance tests for real system applications. At a later point in time, these saving potentials will be addressed in a subsequent publication.

\section{Acknowledgements}

This research was supported by Innosuisse - Swiss Innovation Agency. Special thanks to Tobias Krewer for supporting the manuscript preperation.

\section{Author's contributions}

Conceived the plan: RE, LP, DR; Performed the expeirments: LP, DR; Data analysis: RE, LP, DR; Wrote the paper: RE, DR. Authors have no competing financial interests.

\section{Conflicts of interest}

There are no conflicts to declare.

\section{References}

1. Arruda, E. M.; Boyce, M. C.; J. Mech. Phys. Solids, 1993, 41, 389.

2. Bärtsch, F.; Mayer, T.; Ameli, A.; ASME 2019 Conference on Smart Materials, Adaptive Structures and Intelligent Systems. Proc. SMASIS2019-5671, Louisville, KY, 2019.

3. Bergstrom, J. S.; Boyce, M. C.; Mech. Mater., 2001, 33, 523.

4. Bergstrom, J. S.; Bischoff, J. E.; Int. J. of Structural Changes in Solids, 2010, 2, 31.

5. Bower, D. I.; An Introduction to Polymer Physics; Cambridge University Press, Cambridge, 2002.

6. Brent, R. P.; Algorithms for MinimizationWithout Derivatives; Prentice- Hall, Englewood Cliffs, NJ, 1993.

7. Christensen, R. M.; Theory of Viscoelasticity; An Introduction. Academic Press, New York, 1982.

8. Latorre, M.; Montans, F. J.; Comput. Mech., 2015, 56, 503.

9. Liu, H.; Holzapfel, G. A.; Skallerud, B. H.; Prot, V.; J. Mech. Phys. Solids, 2019, 124, 172.

10. Nelder, J. A.; Mead, R.; Computer J., 1965, 7, 308.

11. Ogden. R. W.; Non-linear Elastic Deformations; Dover Publications, New York, 1997.

12. Shaw, M. T.; MacKnight, W. J.; Introduction to Polymer Viscoelasticity; Wiley-Interscience, New York, 2005.

13. Williams, M. L.; Landel, R. F.; Ferry, J. D.; J. Am. Chem. Soc., 1955, 77, 3701.

14. Zhang, J.; Lu, G.; Ruan, D.; Wang, Z.; Int. J. Mech. Sciences, 2018, 136, 143. 